Análisis de la Variabilidad Genética entre treinta accesiones de tarwi (Lupinus mutabilis Sweet) usando marcadores moleculares ISSR

\title{
Analysis of Genetic Variability among thirty accessions of Andean Lupin (Lupinus mutabilis Sweet) using ISSR molecular markers
}

\author{
Michelle C. Chirinos-Arias ${ }^{1,2,}$; Jorge E. Jiménez ${ }^{3,4}$; Lizbeth S. Vilca-Machaca ${ }^{2}$ \\ ${ }^{1}$ Centro de Diagnóstico Molecular S.A.C., Calle Monterosa 270, oficina 503, Surco, Perú. \\ 2 Facultad de Ciencias, Universidad Nacional Agraria La Molina, Lima, Perú. \\ ${ }^{3}$ Laboratorio de Biotecnología, Programa de Cereales y Granos Nativos, Universidad Nacional Agraria La Molina, Lima, \\ Perú. \\ ${ }^{4}$ Facultad de Agronomía, Universidad Nacional Agraria La Molina, Lima, Perú.
}

Recibido 10 septiembre 2014. Aceptado 14 febrero 2015.

\begin{abstract}
Resumen
Con el fin de realizar el análisis de variabilidad genética inter-accesión de treinta accesiones de tarwi ( $L$. mutabilis Sweet) pertenecientes al Banco de Germoplasma del Instituto Nacional de Innovación Agraria (INIA). Se extrajo el ADN de 300 plantas, se construyeron bulks, se estandarizó el protocolo de amplificación de los marcadores moleculares Inter Simple Sequence Repeat (ISSR), de los cuales se eligió a los más polimórficos y nítidos para corrida en gel de acrilamida. Encontrándose 255 bandas con 8 iniciadores ISSR. El análisis de la variabilidad genética con estos iniciadores comprobó una alta variabilidad genética de las muestras en estudio. Observándose también un polimorfismo relativamente alto para una especie autógama como L. mutabilis. Finalmente los fenogramas mostraron una relación con la ubicación geográfica, posiblemente debido al flujo génico in situ debido al intercambio o venta de semillas en ferias o mercados aledaños a la zona de colecta.
\end{abstract}

Palabras clave: Lupinus mutabilis, bulks, variabilidad genética, ISSR.

\section{Abstract}

In order to make the genetic variability analysis among thirty accessions of andean lupine (L. mutabilis Sweet) belonging to Agrarian Innovation National Institute (INIA) Seed Bank. DNA was extracted from 300 plants and we made bulks. We standardized amplification protocol of Inter Simple Sequence Repeat (ISSR) primers, we chose the most polymorphic primers to run in acrylamide gel. We found 255 ISSR loci with 8 primers. It was found high genetic variability of the samples under study by ISSR markers. Also observed relatively high polymorphism for autogamous species such as andean lupine. Finally phenograms showed a relationship with the geographical location, possibly due to in situ gene flow due to the exchange or sale of seeds in markets near the collection area.

Keywords: Lupinus mutabilis, bulks, genetic variability, ISSR.

\section{Introducción}

Actualmente uno de los problemas más preocupantes es la desnutrición. Existen más de 925 millones de personas desnutridas en el mundo (FAO, 2010a) y el Perú no es ajeno a este problema. Una de las soluciones planteadas es el consumo de alimentos de alto valor nutritivo $\mathrm{y}$ medicinal, especialmente de los granos andinos tal como Lupinus mutabilis Sweet. La importancia de esta leguminosa radica en que fija nitrógeno atmosférico en cantidades apreciables de $100 \mathrm{~kg} / \mathrm{ha}$, restituyendo la fertilidad del suelo (Jacobsen y Mujica, 2006). Además se usa como alimento humano y para animales por su alto contenido de proteínas (41$51 \%)$, aceites (14-24\%), vitaminas y minerales (Castañeda et al., 2008). Sin embargo, su cultivo se da en menor escala por el sabor amargo de las semillas debido a la presencia de alcaloides quinolizidíni-

\footnotetext{
* Autor para correspondencia

E-mail: michelle.christine16@gmail.com (M.C. Chirinos-Arias).
} 
cos (Palacios, 2003; Gross et al., 1988; Ciancio y Mukerji, 2008; Jacobsen y Mujica, 2006; Castañeda et al., 2007; Castañeda et al., 2008; FAO, 2010b).

La búsqueda de especies de importancia para la seguridad alimentaria ha devuelto el interés en estas especies que quedaron relegadas tanto en su cultivo como en la investigación. Lamentablemente en el caso de L. mutabilis solo existe un estudio molecular previo realizado con AFLPs (Jiménez, 2006). Se necesita más investígación para implementar estrategias efectivas para su conservación y mejoramiento, siendo de suma importancia un análisis de variabilidad genética usando otros marcadores moleculares como los Inter Simple Sequence Repeats (ISSR).

Los ISSRs son un tipo de marcador genético dominante que permite obtener los niveles de variación en las regiones microsatélite que se encuentran dispersas en varios genomas, particularmente el nuclear (Zietkiewicz et al., 1994; Rakoczy y Bolibok, 2004). Consisten en un motivo repetido de di - o trinucleótidos complementario a la secuencia del microsatélite (evitando los mononucleótidos que están presentes principalmente en los cloroplastos). En ocasiones es posible agregar a esta secuencia un par de nucleótidos extras arbitrarios en el extremo 3' o en el 5', que jugarán el papel de "anclas", asegurando así que la amplificación inicie siempre en el extremo 5' o en el 3' del microsatélite, respectivamente (Bornet y Branchard, 2001). Las ventajas que ofrece esta técnica se centran principalmente en la alta variación que se detecta, así como en su reproducibilidad en la PCR. Asimismo, no son necesarias altas concentraciones de ADN. Por otro lado, para diseñar los iniciadores no es necesario conocer la secuencia del genoma del organismo en estudio. Pueden visualizarse tanto en geles de agarosa como de acrilamida y son sencillos de montar, rápidos, eficientes y poco costosos (Eguiarte et al., 2007).

El "tarwi" posee amplia diversidad genética. Se han identificado 83 especies del género Lupinus en la región andina de Ecuador, Perú y Bolivia. Presenta gran variabilidad en la arquitectura de la planta, adaptación a suelos, precipitación, temperatura y altura. Así como en el color del grano y la flor (Mujica, 1990).

En las especies de Lupinus del "nuevo mundo" ocurre hibridaciones interespecíficas, lo que también se ha observado en $L$. mutabilis (Camarena et al., 2012). Esto hace que la variabilidad genética de esta planta sea mayor, por lo que es indispensable el estudio de la planta a nivel molecular.

La biotecnología permite incrementar el conocimiento de las especies por medio de la caracterización de los bancos de germoplasma, mediante el uso de marcadores genéticos y particularmente de los marcadores moleculares (Moreno et al., 2004; Azofeifa, 2006) utilizando así la información contenida en el genoma para clasificar material parental y acelerar la selección de las mejores progenies para satisfacer las demandas del mercado que exigen el desarrollo y utilización de cultivares con productividad alta, estable y de cualidades superiores (Ovesná et al., 2002).

Nuestro país posee una amplia biodiversidad que debe ser cuidada y aprovechada de manera racional sobretodo en nuestra situación actual donde, la pérdida de diversidad biológica aumenta día tras día. Es por ello que vale la pena dedicar esfuerzos a la conservación de los recursos genéticos, en particular de aquellos cultivos autóctonos e históricos como el "tarwi" cuyas semillas han sido encontradas en tumbas de la cultura Nazca y representadas en las culturas Tiahuanaco y Chavín (FAO, 2010b).

Bajo estas premisas los objetivos de esta investigación fueron, realizar el análisis de variabilidad genética intergenotípica de treinta accesiones de tarwi (L. mutabilis Sweet) pertenecientes al Banco de Germoplasma del INIA, utilizando marcadores moleculares ISSR e identificar los iniciadores más polimórficos para la población en estudio. 


\section{Materiales y métodos}

\section{Siembra de accesiones}

Se sembraron 10 semillas por maceta, una maceta para cada accesión (300 semillas en total). Las cuales se mantuvieron bajo condiciones de tinglado, se las regó dos a tres veces por semana. Se recolectaron las dos primeras verdaderas hojas a los 15 días de la siembra. Luego se secaron las muestras en sílica gel. Una vez secas, las hojas se trituraron y se colocaron en microtubos de 1,5 $\mathrm{ml}$ para la extracción de ADN. Todos estos procedimientos fueron realizados en condiciones de asepsia, usando en todo momento guantes y alcohol al $70 \%$. La Tabla 1 muestra los datos pasaporte de las 30 accesiones estudiadas en esta investigación.

\section{Extracción y cuantificación del ADN}

Se extrajo el ADN de 300 muestras siguiendo el método micro CTAB (Cetil Trimethyl Ammonium Bromide) indicado por Doyle y Doyle (1987) modificado en el Laboratorio de Biotecnología del Programa de Cereales y Granos Nativos (modificaciones en el tiempo de centrifugación y aumento en el número de veces de lavado en alcohol al 70\%).

Una vez extraído, el ADN se cuantificó en el biofotómetro (Eppendorf) y se realizó una prueba de calidad en geles de agarosa $1 \%$ teñidos con bromuro de etidio.

Tabla 1

Datos pasaporte de 30 accesiones de L. mutabilis estudiados

\begin{tabular}{|c|c|c|c|c|c|c|c|c|}
\hline Código & Accesión & País & Departamento & Provincia & Distrito & $\begin{array}{l}\text { Altitud } \\
(\mathrm{m})\end{array}$ & $\begin{array}{l}\text { Latitud } \\
\text { (S) }\end{array}$ & $\begin{array}{l}\text { Longitud } \\
\text { (W) }\end{array}$ \\
\hline $\mathrm{Ta} 01$ & 3138 & Perú & Cajamarca & Celendín & Celendín & 2950 & $06^{\circ} 46^{\prime} 20^{\prime \prime}$ & $78^{\circ} 06^{\prime} 23^{\prime \prime}$ \\
\hline $\mathrm{Ta} 02$ & 3141 & Perú & Cajamarca & Celendín & Celendín & 2950 & $06^{\circ} 46^{\prime} 20^{\prime \prime}$ & $78^{\circ} 06^{\prime} 23^{\prime \prime}$ \\
\hline $\mathrm{Ta} 03$ & 3142 & Perú & Cajamarca & Celendín & Celendín & 2950 & $06^{\circ} 46^{\prime} 20^{\prime \prime}$ & $78^{\circ} 06^{\prime} 23^{\prime \prime}$ \\
\hline $\mathrm{Ta} 04$ & 3217 & Perú & Cajamarca & Cajamarca & Cajamarca & 2780 & $07^{\circ} 09^{\prime} 12^{\prime \prime}$ & $78^{\circ} 26^{\prime} 33^{\prime \prime}$ \\
\hline $\mathrm{Ta} 05$ & 3214 & Perú & Cajamarca & Cajamarca & Cajamarca & 2780 & $07^{\circ} 09^{\prime} 12^{\prime \prime}$ & $78^{\circ} 26^{\prime} 33^{\prime \prime}$ \\
\hline $\mathrm{Ta} 06$ & 3208 & Perú & Cajamarca & Cajamarca & Cajamarca & 2500 & $07^{\circ} 07^{\prime} 55^{\prime \prime}$ & $78^{\circ} 15^{\prime} 40^{\prime \prime}$ \\
\hline $\mathrm{Ta} 07$ & 3189 & Perú & Cajamarca & Celendín & Celendín & 2950 & $06^{\circ} 46^{\prime} 20^{\prime \prime}$ & $78^{\circ} 06^{\prime} 23^{\prime \prime}$ \\
\hline $\mathrm{Ta} 08$ & 3275 & Perú & Ancash & Huari & Huari & 3150 & $09^{\circ} 20^{\prime} 43^{\prime \prime}$ & $77^{\circ} 10^{\prime} 00^{\prime \prime}$ \\
\hline Ta09 & 3285 & Perú & Cusco & Cusco & Cusco & 3200 & $13^{\circ} 34^{\prime} 54^{\prime \prime}$ & $71^{\circ} 57^{\prime} 33^{\prime \prime}$ \\
\hline Ta10 & 3158 & Perú & Cajamarca & Celendín & Celendín & 2950 & $06^{\circ} 46^{\prime} 20^{\prime \prime}$ & $78^{\circ} 06^{\prime} 23^{\prime \prime}$ \\
\hline Ta11 & 3163 & Perú & Cajamarca & Celendín & Celendín & 2950 & $06^{\circ} 46^{\prime} 20^{\prime \prime}$ & $78^{\circ} 06^{\prime} 23^{\prime \prime}$ \\
\hline Ta12 & 4022 & Perú & Cajamarca & San Marcos & Obeco & 3000 & $07^{\circ} 16^{\prime} 11^{\prime \prime}$ & $78^{\circ} 05^{\prime} 18^{\prime \prime}$ \\
\hline Ta13 & 3330 & Perú & Cajamarca & Cajabamba & Colcabamba & 2600 & $07^{\circ} 35^{\prime} 11^{\prime \prime}$ & $78^{\circ} 06^{\prime} 55^{\prime \prime}$ \\
\hline Ta14 & 3335 & Perú & Cajamarca & Cajabamba & Cajabamba & 2500 & $07^{\circ} 07^{\prime} 55^{\prime \prime}$ & $78^{\circ} 15^{\prime} 40^{\prime \prime}$ \\
\hline Ta15 & 3342 & Perú & La Libertad & $\begin{array}{l}\text { Sánchez } \\
\text { Carrión }\end{array}$ & Kayra & 3350 & $07^{\circ} 34^{\prime} 49^{\prime \prime}$ & $78^{\circ} 03^{\prime} 59^{\prime \prime}$ \\
\hline Ta16 & 4878 & Perú & Amazonas & Chachapoyas & Leymebamba & 2550 & $06^{\circ} 31^{\prime} 44^{\prime \prime}$ & $77^{\circ} 38^{\prime} 02^{\prime \prime}$ \\
\hline Ta17 & 3462 & Perú & Cajamarca & Cajabamba & $\begin{array}{l}\text { Piedra } \\
\text { Grande }\end{array}$ & 2800 & $07^{\circ} 35^{\prime} 50^{\prime \prime}$ & $77^{\circ} 55^{\prime} 21^{\prime \prime}$ \\
\hline Ta18 & 4371 & Perú & Cajamarca & San pablo & Santa Rosa & 2800 & $06^{\circ} 50^{\prime} 51^{\prime \prime}$ & $78^{\circ} 45^{\prime} 16^{\prime \prime}$ \\
\hline Ta19 & 4043 & Perú & Cajamarca & Celendín & Huasmin & 3100 & $06^{\circ} 45^{\prime} 08^{\prime \prime}$ & $78^{\circ} 10^{\prime} 25^{\prime \prime}$ \\
\hline Ta20 & 4009 & Perú & Cajamarca & Cajabamba & Lluchubamba & 3010 & $07^{\circ} 26^{\prime} 49^{\prime \prime}$ & $77^{\circ} 51^{\prime} 14^{\prime \prime}$ \\
\hline $\mathrm{Ta} 21$ & 6146 & Perú & Cajamarca & Celendín & José Galvez & 2850 & $06^{\circ} 42^{\prime} 45^{\prime \prime}$ & $78^{\circ} 12^{\prime} 45^{\prime \prime}$ \\
\hline Ta22 & 6143 & Perú & Cajamarca & Celendín & José Galvez & 2850 & $06^{\circ} 42^{\prime} 45^{\prime \prime}$ & $78^{\circ} 12^{\prime} 45^{\prime \prime}$ \\
\hline Ta23 & - & - & - & - & - & - & - & - \\
\hline $\mathrm{Ta} 24$ & 6136 & Perú & Cajamarca & Celendín & Quillimbash & 2850 & $06^{\circ} 47^{\prime} 55^{\prime \prime}$ & $78^{\circ} 09^{\prime} 29^{\prime \prime}$ \\
\hline Ta25 & 6236 & Perú & Cajamarca & Cajabamba & Mercado & 2654 & $07^{\circ} 35^{\prime} 22^{\prime \prime}$ & $78^{\circ} 01^{\prime} 52^{\prime \prime}$ \\
\hline Ta26 & 5919 & Perú & Cajamarca & Hualgayoc & Shitamayo & 3100 & $06^{\circ} 14^{\prime} 33^{\prime \prime}$ & $78^{\circ} 36^{\prime} 26^{\prime \prime}$ \\
\hline Тa27 & 5394 & $\begin{array}{c}\text { Boliv } \\
\text { ia }\end{array}$ & - & - & - & 2830 & $16^{\circ} 43^{\prime} 00^{\prime \prime}$ & $67^{\circ} 02^{\prime} 00^{\prime \prime}$ \\
\hline Тa28 & 5393 & Perú & Cajamarca & Chota & La Cultura & 3260 & $06^{\circ} 37^{\prime} 03$ '” & $78^{\circ} 36^{\prime} 21^{\prime \prime}$ \\
\hline Тa29 & 6338 & Perú & Puno & Yunguyo & Yunguyo & 3800 & $16^{\circ} 15^{\prime} 38^{\prime \prime}$ & $69^{\circ} 05^{\prime} 37^{\prime \prime}$ \\
\hline Тa30 & 3329 & Perú & Cajamarca & Cajabamba & Colcabamba & 2600 & $07^{\circ} 35^{\prime} 11^{\prime \prime}$ & $78^{\circ} 06^{\prime} 55^{\prime \prime}$ \\
\hline
\end{tabular}




\section{Amplificación del ADN}

Las muestras de ADN se diluyeron a una concentración uniforme de $10 \mathrm{ng} / \mu \mathrm{l}$ con agua ultrapura. Luego se creó un bulk para cada una de las 30 accesiones para lo cual se tomó $2 \mu \mathrm{l}$ de cada una de las 10 muestras diluidas de cada accesión, obteniéndose una concentración final de 10 ng/ $\mu 1$ para el análisis inter-accesión. El mix de PCR estuvo compuesto por ADN (10 ng/reacción), iniciadores de intersecuencia (1 $\mu \mathrm{M}), \mathrm{MgCL}_{2}(4 \mathrm{mM})$, dNTP $(2 \mathrm{mM})$, Taq DNA Polimerasa Fermentas (1 U), buffer Taq $(1 \mathrm{x})$ y completando con agua grado PCR para un volumen final de 10 $\mathrm{uL}$. Las condiciones de PCR fueron de 94 ${ }^{\circ} \mathrm{C}$ por $3 \mathrm{~min}$, seguido de 39 ciclos de 94 ${ }^{\circ} \mathrm{C}$ por 30 segundos, $48{ }^{\circ} \mathrm{C}$ por 45 s y $72{ }^{\circ} \mathrm{C}$ por 2 minutos, con una amplificación final de $72{ }^{\circ} \mathrm{C}$ por $10 \mathrm{~min}$.

\section{Post PCR}

Para el tamizaje de iniciadores se emplearon geles de agarosa al 1,5\%, se seleccionaron a los iniciadores que mayor amplificaron y que mostraron más polimorfismo para corrida vertical en gel de acrilamida- Bis acrilamida (19:1) al 6\%.

Para visualizar el patrón de bandas característico para cada iniciador y con esto la presencia o ausencia de fragmentos de ADN (polimorfismos) fue necesario usar el método de electroforesis en gel de acrilamida y la posterior tinción, fijación y revelado con nitrato de plata.

Por último para corroborar que las lecturas de los bulks fueron correctas y estuvieran entre $300 \mathrm{bp}$ y $3000 \mathrm{bp}$, se tomaron tres accesiones al azar Ta05, Ta07 y Ta09. Cada una conformada por 10 individuos de la misma accesión, haciendo un total de 30 muestras (cuyo ADN se extrajo y se conservó por separado a $-20{ }^{\circ} \mathrm{C}$ ). De esta manera también se pudo estimar el nivel de variación de estas accesiones.

\section{Análisis de datos}

Los productos de la amplificación fueron anotados en una matriz de datos binaria, se colocó el valor de " 1 " si hay presencia de banda y "0" si hay ausencia de banda. Se consideraron sólo aquellas bandas que presentaron una intensidad uniforme, aquellas que fueron borrosas o muy tenues no se consideraron para evitar errores.

El peso de cada amplicón (bp) se determinó por comparación con el marcador de peso GeneRuler ${ }^{\mathrm{TM}} 100$ bp Plus DNA Ladder. Para el caso de ISSR lectura de las bandas usualmente es entre $400 \mathrm{bp}$ a $3000 \mathrm{bp}$. Aunque en algunos casos es de 900 bp a 300 bp. La lectura nos permitió construir la matriz básica de datos y determinar el total de bandas para cada iniciador. Así como las bandas polimórficas, monomórficas y el porcentaje de polimorfismo (P\%).

Se estableció cada accesión (bulk) como una unidad taxonómica operativa (OTU). Para agrupar cada OTU de acuerdo al grado de similitud, se hizo un análisis por pares de OTU usando ligamientos promedios o UPGMA (Unweighted Pair Group of Arithmetic Mean), seguido de un método de agrupamiento secuencial, aglomerativo, jerárquico y anidado (SAHN) para formar un fenograma se escogió el índice de similitud de Jaccard. Se eligió este índice de similitud, muy utilizado en estudios de variabilidad genética en el género Lupinus, ya que en los datos de presencia/ausencia, un criterio válido es considerar que dos muestras son más parecidas cuantos más unos (bandas presentes) compartan, por lo que la coincidencia de ceros (bandas ausentes) no aporta a la similitud ya que puede estar generada por falta de información. Este paso fue posible mediante el uso del software especializado NTSYSpc (Numerical Taxonomy System for personal computer, version 2.0) disponible en el Programa de Cereales y Granos Nativos de la Universidad Nacional Agraria La Molina (UNALM), Lima-Perú.

Adicionalmente se usó el software Google Earth, en el cual se identificaron geográficamente las accesiones en estudio según sus coordenadas, se evalúo los perfiles de elevación y distancias entre las accesiones, facilitando una mejor interpretación de los resultados. 
Para el análisis intragenotípico de las tres accesiones escogidas al azar, se calculó el Contenido de Índice Polimórfico (PIC), descrito por Vuylsteke et al. (2000). Este valor evalúa la informatividad de un marcador en la población de acuerdo a las frecuencias de los alelos (Weising et al., 2005). También se calculó los valores $\mathrm{P} \%$ para cada iniciador y sus valores promedio.

\section{Resultados y discusión}

\section{Iniciadores ISSR para el análisis de los} 30 bulks de las accesiones de $L$. mutabilis De un total de 65 iniciadores ISSR se eligieron 8 iniciadores, los más nítidos y polimórficos según los geles de agarosa $(1,5 \%)$ para corrida en gel de acrilamida. Los iniciadores elegidos fueron los siguientes (ver Tabla 2): 811 (e.g. Jian et al., 2004; Su et al., 2008; Zhu et al., 2011; Shafie et al., 2011), 834 (e.g. Zhao et al., 2005; Svetleva et al., 2006; Nascimiento et al., 2010; Shafie et al., 2011), 835 (e.g. Joshi et al., 2000; Jian et al., 2004; Guo et al., 2009), 841 (e.g. Cao et al., 2006; Kothera et al., 2007; Aguilera et al., 2011; Shafie et al., 2011), 842 (e.g. Su et al., 2008; Guo et al., 2009), 889 (e.g. Hatcher et al., 2004; Lázaro y Aguinagalde, 2006; Wang et al., 2008), 891 (e.g. Lázaro y Aguinagalde, 2006; Roh et al., 2007; Wang et al., 2008) y BOR5 (e.g. Sbabou et $a l ., 2010)$. De ellos el iniciador 841 y 842 (Talhinhas et al., 2003) y el iniciador BOR5 (Sbabou et al., 2010), han sido efectivamente utilizados en especies del género Lupinus. Mientras que, los iniciadores 841,834 y 811 fueron utilizados en diversos cultivos.

Los iniciadores 884 (e.g. Isshiki et al., 2008) y 890 (e.g. Hatcher et al., 2004; Lázaro y Aguinagalde, 2006; Kothera et al., 2007) mostraron polimorfismo en el gel de agarosa $(1,5 \%)$, aunque muy poco, pero debido a su nitidez se los corrió en geles de poliacrilamida. Sin embargo, mostraron escaso polimorfismo $20 \%, 83 \%$ y $10 \%$, respectivamente, por lo que no se los incluyó en este análisis.

Los iniciadores que poseen las repeticiones
$(\mathrm{AG})_{8}$ y $(\mathrm{GA})_{8}$ con o sin anclajes 5'o 3'. Así como los que tienen motivos $(\mathrm{AG})_{7}$, $(\mathrm{AC})_{7},(\mathrm{GT})_{8}$ y $(\mathrm{TG})_{7}$ tuvieron un alto grado de polimorfismo y abundancia en el genoma de L. mutabilis lo que coincide con lo encontrado por Shafie et al. (2011) y Sbabou et al. (2010) en el estudio de diferentes especies del género Lupinus como $L$. albus, $L$ angustifolius, $L$. consetinii y L. luteus.

\section{Tabla 2}

Secuencia y motivos de los iniciadores más polimórficos

\begin{tabular}{ccl}
\hline \hline Iniciador & motivo & \multicolumn{1}{c}{ Secuencia } \\
\hline 834 & $(\mathrm{AG})_{8} \mathrm{YT}$ & AGAGAGAGAGAGAGAGYT \\
BOR 5 & $(\mathrm{AG})_{8}$ & AGAGAGAGAGAGAGAG \\
841 & $(\mathrm{GA})_{8} \mathrm{YC}$ & GAGAGAGAGAGAGAGAYC \\
842 & $(\mathrm{GA})_{8} \mathrm{YG}$ & GAGAGAGAGAGAGAGAYG \\
811 & $(\mathrm{GA})_{8} \mathrm{C}$ & GAGAGAGAGAGAGAGAC \\
889 & DBD $(\mathrm{AC})_{7}$ & DBDACACACACACACAC \\
891 & $\mathrm{HVH}(\mathrm{TG})_{9}$ & HVHTGTGTGTGTGTGTGTGTG \\
835 & $(\mathrm{AG})_{8} \mathrm{YC}$ & AGAGAGAGAGAGAGAGYC \\
\hline
\end{tabular}

El iniciador $810\left((\mathrm{GA})_{8} \mathrm{~T}\right), 811\left((\mathrm{GA})_{8} \mathrm{C}\right)$ y $812\left((\mathrm{GA})_{8} \mathrm{~A}\right)$ presentan similar motivo $\left((\mathrm{GA})_{8}\right)$. Sin embargo solo los iniciadores 811 y 812 amplifican. De estos solo el primero es más polimórfico y nítido. Esto se debería a que en los bulks estudiados, el microsatélite $(\mathrm{GA})_{8}$ estaría flanqueado en el extremo 3' por "C" o "A" y no por " $T$ ".

Se descartó iniciadores con patrón de bandas poco nítido, como es el caso del iniciador BOR $7(\mathrm{AC})_{8}$. Así como de aquellos iniciadores con repeticiones TC (822) y CT $(813,815,822$ y 844), por presentar este mismo problema de presencia de barridos. Según Bornet y Branchard (2001) la presencia de barridos se puede disminuir aumentando la temperatura de hibridación al momento de la PCR, ya que esta temperatura es uno de los factores principales que determinan la calidad de las bandas en el gel (Astarini et al., 2006). Este problema es usual en iniciadores no anclados (Joshi et al. 2000).

\section{Iniciadores ISSR más informativos y polimórficos}

Se analizaron un total de 255 bandas con 8 iniciadores para los 30 bulks. Según 
Hollingsworth y Ennos (2004), este número de bandas analizadas está en el rango recomendado para el estudio de variabilidad genética usando marcadores dominantes, como es el caso de los ISSR. Un valor representativo, a comparación del trabajo de Konate et al. (2009) que realizó un estudio intergenotípico (mediante el uso de bulks) con 10 accesiones en algarrobo, en el que encontró 176 bandas con 16 iniciadores. Mientras que, en el trabajo de Sica et al. (2005) reportaron 228 bandas con 23 iniciadores.

El porcentaje de polimorfismo promedio de los 30 bulks fue de 58,82\%. Siendo $40 \%$ el valor más bajo, correspondiente al iniciador 889 y 77,5\% el valor más alto, correspondiente al iniciador 834. Mientras que, el monomorfismo promedio fue de $41,18 \%$, valores que se encuentran en el rango aceptado según Bussell et al. (2005), que señala un porcentaje mínimo de monomorfismo de $20 \%$ para este tipo de estudios. Aunque mayor al reportado por Konate et al. (2009) en su estudio de 10 bulks de la especie alógama algarrobo, donde reportó un porcentaje de monomorfismo de $22,73 \%$. Ver todos los valores en la Tabla 3.

La variabilidad genética del cultivo de $L$. mutabilis expresada en porcentaje de bandas polimórficas $(58,82 \%)$ refleja su sistema de polinización autógamo pero con un considerable grado de alogamia (Sevilla y Holle, 2004) lo que es común en especies de Lupinus (Horovitz y Harding, 1983). Sistema mixto que le proporciona mayor diversidad comparada con plantas autógamas cuyo valor de polimorfismo oscila entre 34\% (e.g. Amaranthus tricolor: $\mathrm{Xu}$ y Sun, 2001), 28\% (e.g. arroz: Girma et al., 2010) pero menor con respecto a especies alógamas cuyo valor oscila entre 85,2\% (eg. rabanito: Liu et al., 2008), 70,77\% (e.g. coliflor: Astarini et al., 2006), etc., menor a $50 \%$ de polimorfismo.

También se evidencia que el iniciador 834 produjo mayor polimorfismo $(77,50 \%)$ que el mostrado por Venkatachalam et al. (2008) en banano (38,46\%). Así como, el iniciador 835 presentó un polimorfismo de $61,36 \%$. Mayor que el observado en tomate en el trabajo de Aguilera et al. (2011) $(22,22 \%)$.

Se muestra que el iniciador 889 es el que presenta menor porcentaje de polimorfismo de la población en estudio (40\%). Sin embargo al comparar este valor con otros estudios en diferentes plantas y con la misma temperatura de hibridación (Ta). Se observa que es más polimórfico que el reportado por Aguilera et al. (2011) en tomate $(\mathrm{P}=15,38 \%)$. Aunque menos polimórfico que el reportado por Wang et al. (2008) en la planta china yiaogulan (orquídea de la vid enroscada) con un $100 \%$ de polimorfismo.

Es importante señalar "la misma temperatura de hibridación" en el párrafo anterior, ya que la mayor riqueza de los patrones de bandas en iniciadores no anclados está determinada por la alta "Ta", que debe ser mucho mayor a la temperatura de fusión (Tm) pero no tan restrictiva como para inhibir la amplificación (Fisher et al., 1996). Por tanto diferentes "Ta" pueden dar patrones de banda diferentes.

\section{Tabla 3}

Polimorfismo de productos de amplificación de los 30 bulks de L. mutabilis. Donde \%P: porcentaje de loci polimórfico

\begin{tabular}{|c|c|c|c|c|c|c|c|c|c|c|c|}
\hline \multicolumn{3}{|c|}{ Accesiones } & $\begin{array}{c}811 \\
3000 \leq x \geq \\
900 \mathrm{pb}\end{array}$ & $\begin{array}{c}834 \\
3000 \leq \mathrm{x} \geq \\
400 \mathrm{pb}\end{array}$ & $\begin{array}{c}835 \\
3000 \leq x \geq \\
400 \mathrm{pb}\end{array}$ & $\begin{array}{c}841 \\
3000 \leq x \geq \\
600 \mathrm{pb}\end{array}$ & $\begin{array}{c}842 \\
3000 \leq x \geq \\
300 \mathrm{pb}\end{array}$ & $\begin{array}{c}889 \\
3000 \leq \mathrm{x} \geq \\
900 \mathrm{pb}\end{array}$ & $\begin{array}{c}891 \\
3000 \leq \mathrm{x} \geq \\
400 \mathrm{pb}\end{array}$ & $\begin{array}{c}\text { Bor5 } \\
3000 \leq \mathrm{x} \geq \\
500 \mathrm{pb}\end{array}$ & Total \\
\hline \multicolumn{3}{|c|}{$\mathrm{N}^{\circ}$ de bandas } & 18 & 40 & 44 & 37 & 40 & 20 & 36 & 20 & 255 \\
\hline \multirow{2}{*}{$\begin{array}{c}\text { Ta } \\
1-30\end{array}$} & Bandas & $\mathrm{N}^{\circ}$ & 10 & 31 & 27 & 20 & 21 & 8 & 22 & 11 & 150 \\
\hline & polimórficas & $\% \mathrm{P}$ & 55,56 & 77,50 & 61,36 & 54,05 & 52,50 & 40,00 & 61,00 & 55,00 & 58,82 \\
\hline
\end{tabular}


De aquí que sea posible que aumentando la temperatura de hibridación del iniciador 890 , este hubiera dado mayor polimorfismo y no solo un $10 \%$. Según Isshiki et al. (2008) este iniciador presentó 100\% de polimorfismo en berenjena, pero a una temperatura de annealing de $58{ }^{\circ} \mathrm{C}$, es decir más alta que la nuestra. Por lo que hay que tener en cuenta estos datos para estudios posteriores en L. mutabilis.

El alto grado de polimorfismo se puede explicar (en el caso del tarwi) por "el color de la testa" ya que es un indicio de polinización cruzada (Horovitz y Harding,
1983) y las accesiones estudiadas presentan colores muy variados. Aunque no se debe descartar el flujo de material genético entre las accesiones debido a que se encuentran muy cercanas.

Variabilidad genética intergenotípica de las 30 accesiones de $L$. mutabilis

El fenograma construido basado en el UPGMA (Figura 1) muestra que las accesiones en estudio pueden ser divididas en dos grupos (indicados en números romanos).
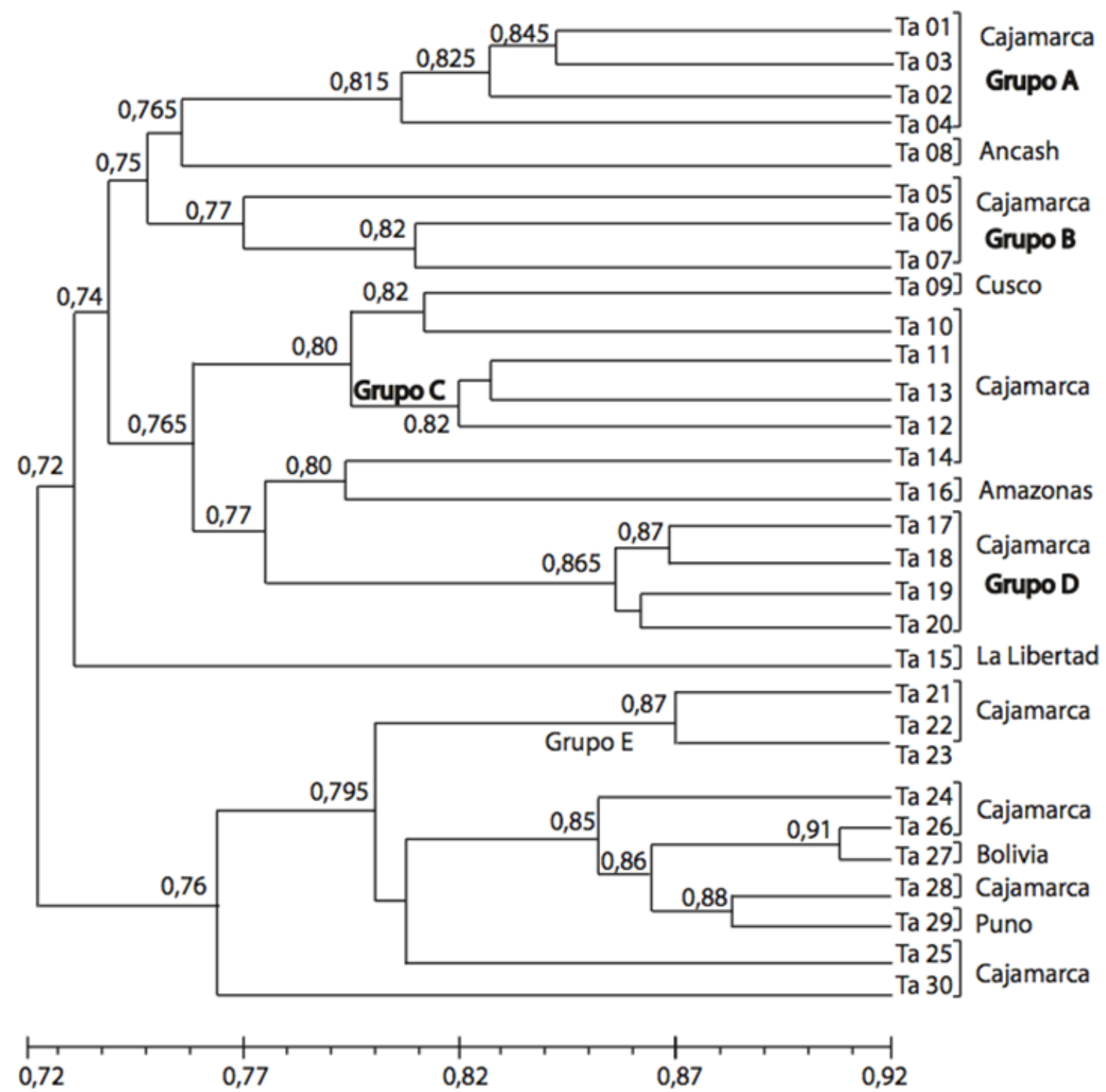

Coeficiente

Figura 1. Fenograma de las 30 accesiones de L. mutabilis analizadas por marcadores ISSR: basado en el análisis de similitud genética (coeficiente Jaccard) utilizando 255 bandas. En números romanos se detallan los principales grupos. 
El grupo I se subdivide en Ia y Ib. El grupo Ia se subdivide en Ia1 y Ib1 $(\mathrm{J}=0,795)$. En el primero se observa el grupo $\mathrm{E}(\mathrm{J}=0,87)$, todos pertenecientes a Cajamarca. Además dentro de la segunda rama se tiene a las accesiones Ta $27(\mathrm{~J}=0,91)$ y Ta $29(\mathrm{~J}=0,88)$ que corresponden a Bolivia y Puno respectivamente. Lo cual es de esperar debido a la cercanía de estas zonas en el altiplano. Mientras que, en el Ib tenemos a la accesión Ta30 $(\mathrm{J}=0,76)$, perteneciente a Cajamarca, pero que se encuentra separada del resto.

Dentro del grupo II tenemos la subdivisión IIa y IIb $(\mathrm{J}=0,73)$. La primera se subdivide en IIa1 y IIa2. En IIa1 se observa al grupo A $(\mathrm{J}=0,815)$, donde todas accesiones pertenecen a Cajamarca, junto a la accesión Ta $08(\mathrm{~J}=0,765)$, que pertenece a Ancash. Sin embargo esto se puede explicar por la cercanía del departamento de Ancash con el de Cajamarca. En esta rama (IIa1) se observa un grupo $\mathrm{B}(\mathrm{J}=0,77)$ muy claro y cuyas accesiones corresponden en su totalidad al departamento de Cajamarca. Por otro lado el subgrupo IIa2 es más heterogéneo y se divide en IIa2.1 y IIa2.2. El primero tiene una accesión de Cusco $(\mathrm{Ta} 09, \mathrm{~J}=0,82)$ y el grupo $\mathrm{C}(\mathrm{J}=$ 0,83 ) de Cajamarca. Mientras que, el segundo presenta al grupo $\mathrm{D}(\mathrm{J}=0,865)$, también de Cajamarca. Aunque también se observa a la accesión Ta $16(\mathrm{~J}=0,80)$ de Amazonas. Esto también se puede explicar por la cercanía de Amazonas con Cajamarca. Finalmente el subgrupo IIb tiene solo una accesión que corresponde a La Libertad (Ta15), al ser la única accesión de este lugar es de esperar que se encuentre separada, aunque unida más adelante por accesiones de Cajamarca, por encontrarse en lugares geográficamente cercanos.

Como se puede apreciar esta clasificación guarda una alta relación con la distribución geográfica, cuando se compara el lugar de donde provienen las accesiones según sus datos pasaportes de departamento y distrito (Tabla 1) con los datos del fenograma (Figura 1).

Los departamentos de Cajamarca, La
Libertad y Amazonas se encuentran muy cercanos. Al igual que Cusco, Puno y Bolivia. Estos datos concuerdan con el fenograma, ya que las accesiones de Bolivia y Puno se encuentran en ramas cercanas y con un índice de similitud similar. Sin embargo, la accesión de Cusco, se acerca más a las de Cajamarca. A pesar de pertenecer a lugar geográficos distintos y no muy cercanos.

Todos los datos de ubicación geográfica (departamento y provincia) coinciden con los de las coordenadas. Sin embargo observamos que la accesión Ta15, según las coordenadas provistas por los datos pasaporte, no se encuentra en el departamento de La Libertad, sino en el departamento de Cajamarca, provincia de Cajabamba. Por lo que en el fenograma su accesión debería ser más cercana a las de Cajamarca. Lo que no ocurre en este estudio. Es más la accesión Ta 15 tiene una similitud de 0,73 , por lo que se separa tempranamente de las de Cajamarca en el grupo IIb.

Google Earth nos permitió apreciar que las accesiones más cercanas a la accesión 15 son Ta 25 por el este y Ta 13 con Ta 30 por el oeste. Entre la Ta25 y la accesión Ta15 hay una distancia de 4,09 km (Figura 2). Mientras que Ta13 y Ta30 distan 5,47 $\mathrm{km}$ de Ta15 (Figura 3). Por otro lado, el perfil de elevación nos permite predecir cómo se da probablemente el flujo de polen ya que la altitud es una barrera geográfica que nos ayuda a decir que el flujo de polen se da de mayor a menor altitud. Aunque son necesarios más estudios sobretodo de dirección de viento. En cuanto a su perfil de elevación se observa que Ta 25 se encuentra más elevado geográficamente que Ta15 (aproximadamente $3247 \mathrm{~m}$ y $2670 \mathrm{~m}$ respectivamente, según la Figura 2). Por tanto el flujo de polinización probablemente se daría a Ta25 y Ta15 (Figura 2 recuadro de abajo). Por otro lado, como se observa en el fenograma esto no ocurre, pues Ta 15 se encuentra en el grupo I; mientras que Ta 25 en el grupo II. 
Del mismo modo Ta15 se encuentra más elevado geográficamente que Ta 13 y Ta 30 (aproximadamente $2670 \mathrm{~m}$ y $2347 \mathrm{~m}$ respectivamente, según la Figura 3), por lo que la polinización probablemente se daría de Ta15 hacia Ta13 y Ta 30. Esto es más probable para las accesiones Ta 13 y Ta 15 ya que según el fenograma, estas accesiones se encuentran en el grupo II. Lo que no ocurre entre Ta13 y Ta30.

Por otro lado al comparar Ta 15 con la accesión más cercana por el norte (Ta 12). Se observa que Ta 12 está más elevado que Ta 15 (3579 m y $2670 \mathrm{~m}$ ). Además que la distancia que las separa es mucho mayor que las anteriores $(35,8 \mathrm{~km})$. Sin embargo ambas se encuentran en el grupo I (Figura 4). Otra explicación es que los datos de las coordenadas de Ta15 no sean los correctos, ya que según el fenograma (Figura 1), esta accesión está en una rama separada que, si bien es cercana a las de Cajamarca, es la única que forma el grupo IIb. Sin embargo, son necesarios más estudios.

Por otro lado, la accesión de Puno (Ta29) y la de Bolivia (Ta27) se encuentran muy cercanas en el fenograma (grupo Ib1). Esto se puede deber a la cercanía geográfica $(234 \mathrm{~km})$. Además que el perfil de elevación prácticamente se mantiene de 3 $866 \mathrm{~m}$ en Perú hasta $2804 \mathrm{~m}$ en Bolivia. Aunque se presenta un punto más elevado cercano a Ta 27 de $5440 \mathrm{~m}$, pero que no pareciera influir en el flujo de polen.

Este análisis sugiere flujo genético por migración de polen debido a condiciones geográficas cercanas que se corroboran mediante el fenograma. Debido a que $L$. mutabilis es una especie predominantemente autógama (Sevilla y Holle, 2004), instituciones como CIMMYT (Centro Internacional de Mejoramiento de Maíz y Trigo), CIAT (Centro Internacional de Agricultura Tropical) regeneran las accesiones que tienen en sus bancos de germoplasma. Sin embargo, parece no ser lo más adecuado ya que muchas veces la separación de semillas se da por observaciones fenotípicas directas como es el caso del Programa de Cereales y Granos Nativos, aumentando el porcentaje de error, debido a la combinación de colores de semilla entre las accesiones antes de formar el bulk.

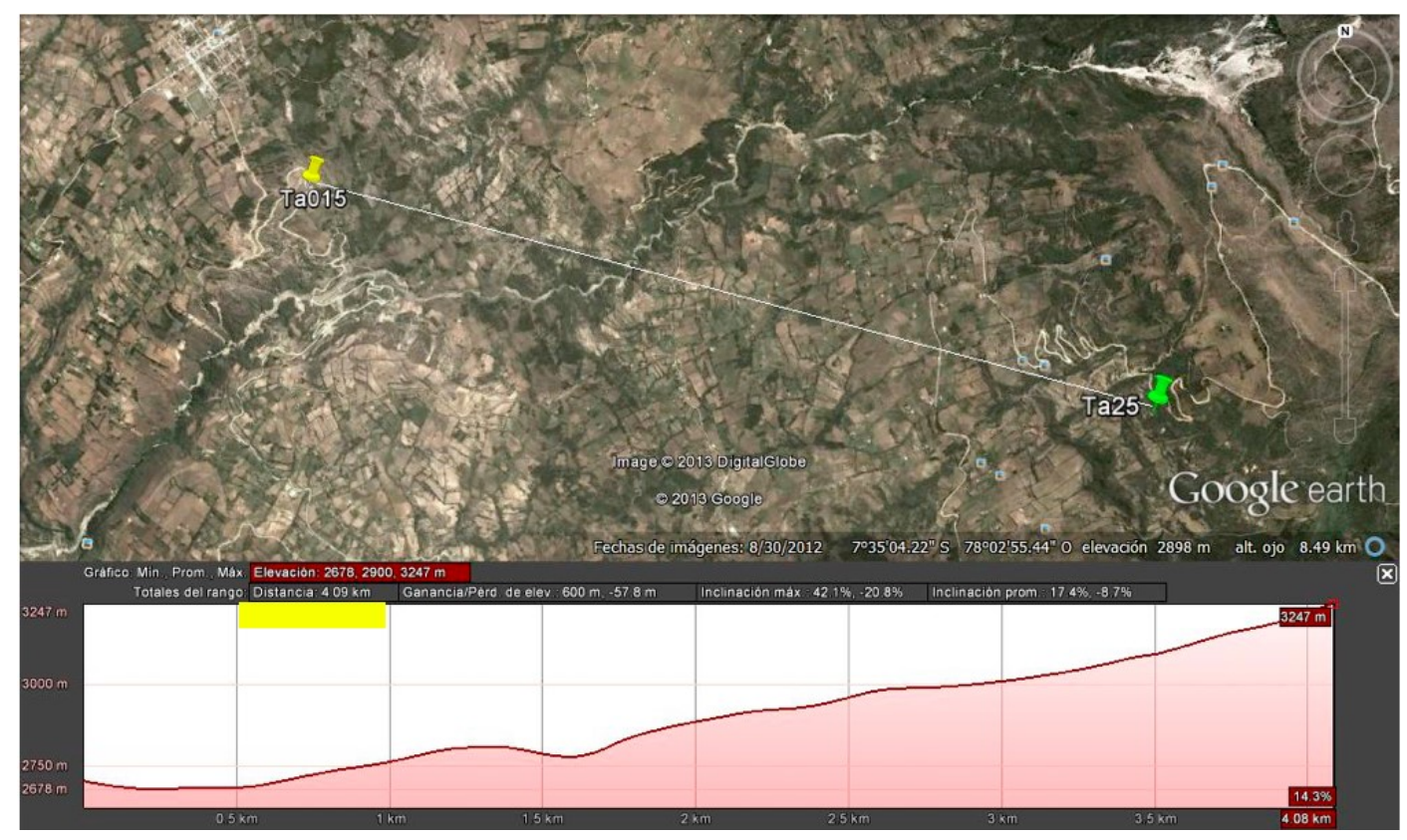

Figura 2. Perfil de elevación entre la accesión más cercana por el este (Ta25). La línea gruesa muestra la distancia en Kilómetros entre las accesiones. 


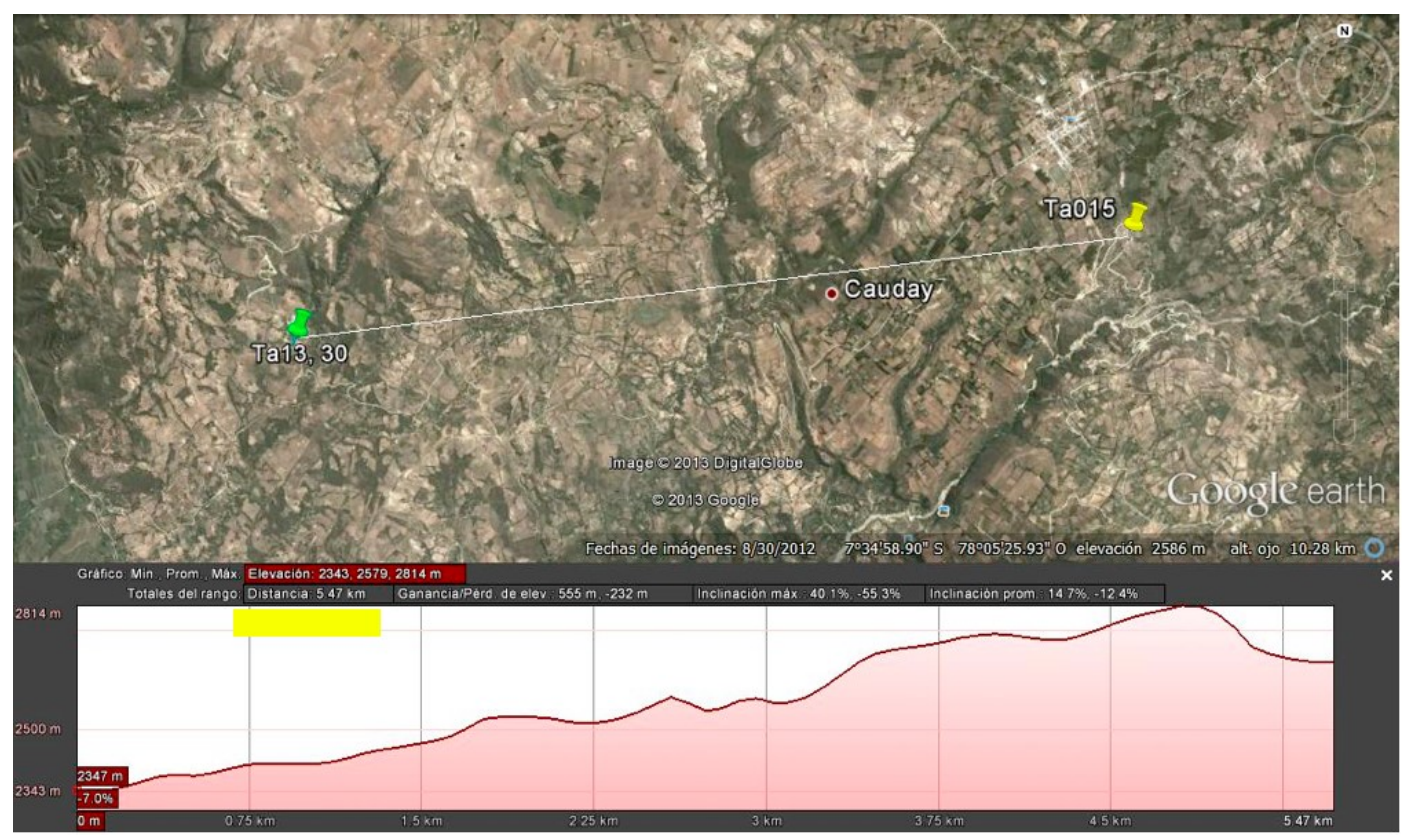

Figura 3. Perfil de elevación entre la accesión más cercana por oeste (Ta13 y Ta 30) de Ta15, todas pertenecientes al Departamento de Cajamarca. La línea gruesa muestra la distancia en Kilómetros entre las accesiones.

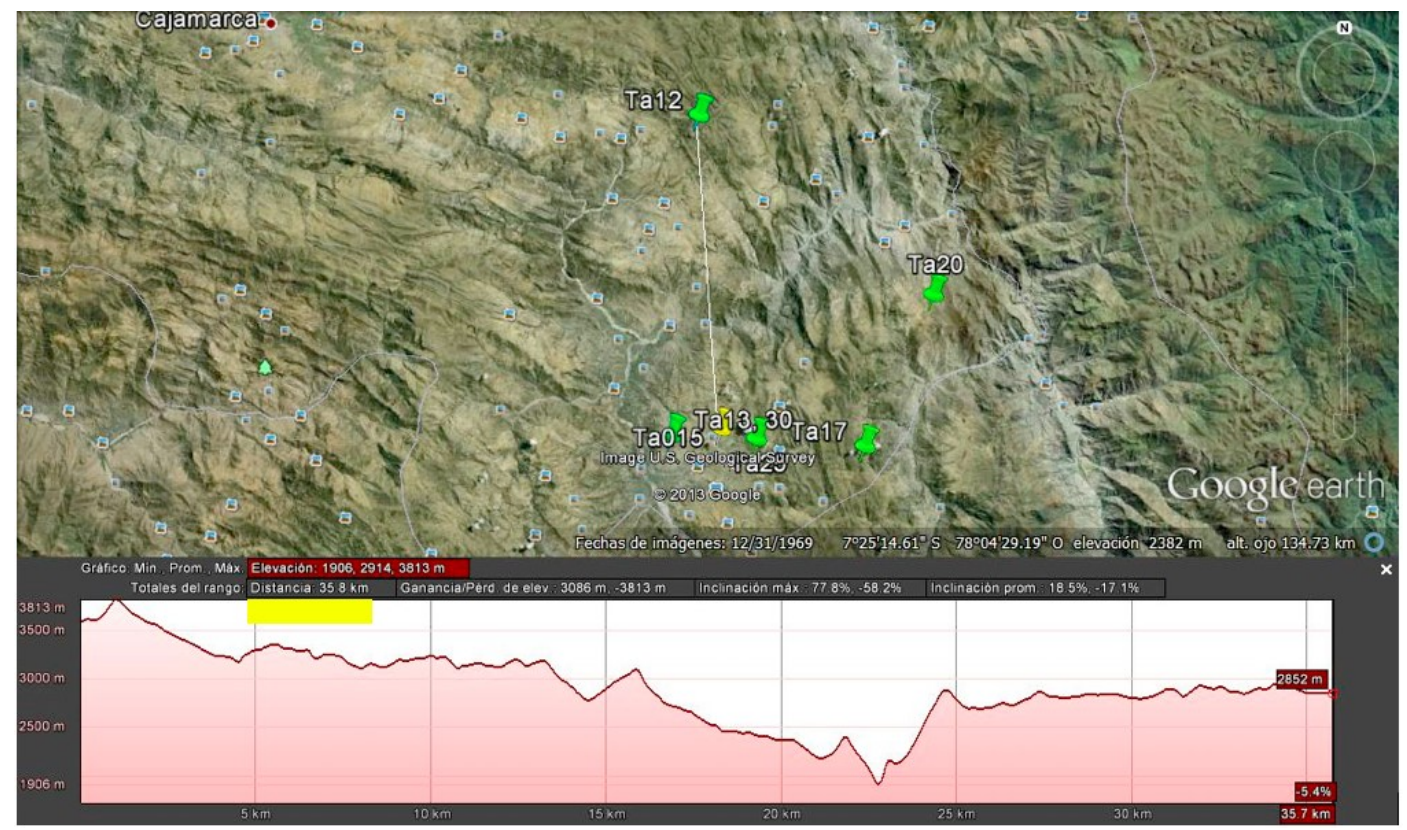

Figura 4. Perfil de elevación entre la accesión más cercana por el norte (Ta25) a Ta15. La línea amarilla muestra la distancia en kilómetros entre las accesiones.

Además L. mutabilis al ser una especie autógama puede formar multilíneas, por lo que el bulk puede ser afectado. Por otro lado, la alta variabilidad genética también se puede explicar por posibles polinizadores (insectos) de pequeño tamaño, debido a la estructura floral. Además el intercambio de material por parte de los agricultores en ferias aledañas puede también contribuir a explicar estos resultados. El número de individuos que conforman el bulk podría afectar el 
análisis. Aunque estos estudios son usados para obtener información de la variabilidad genética entre diferentes poblaciones (Loarce et al., 1996), los alelos con menor frecuencia, podrían no estar representados. Por lo que se dice que este tipo de análisis interge-notípico es menos informativo y que usualmente se representa solo a los alelos más frecuentes en la población (Ferriol et al., 2001). Sin embargo, en esta investí-gación se han usado 10 muestras por bulk. Lo que coincide con lo recomendado por Gilbert et al. (1999) donde señala que los análisis intergenotípicos son confiables con grandes números de muestras. Por ejemplo Yang y Quiros (1993) y Fikiru et al. (2007) demostraron que bulks formados de $10,20,30,40$ y 50 individuos son confiables y que incluso resultan en el mismo perfil genético que el obtenido con un análisis intragenotípico (intra-accesión) cuando se usa marcadores RAPD. Además Hou et al. (2005) señala que para un análisis de bulks exitoso, es necesario un mínimo de 10 individuos como se corroboró en un estudio en cebada usando tanto RAPD como ISSR.

\section{Análisis intra-accesión de 3 accesiones de $L$. mutabilis \\ Las lecturas de los geles procedentes de las muestras de ADN de los 10 individuos de las accesiones tomadas al azar, confirman los resultados obtenidos mediante el}

análisis de los bulks. El menor PIC promedio $(0,31)$ corresponde al iniciador 891 y el mayor valor $(0,40)$ corresponde al iniciador 834 (que presenta mayor eficiencia para detectar bandas polimórficas) (Tabla 4). Todos los valores observados son menores a 0,5 , como ocurre con otros marcadores dominantes (Jiménez, 2006). El máximo valor de PIC para marcadores de este tipo es 0,5; mientras que, para codominantes es de 1 . El PIC hace referencia al valor del marcador para detectar polimorfismo en la población. Como se aprecia depende del número de alelos detectables y la distribución de su frecuencia y su equivalente a la diversidad genética (Muthusamy et al., 2008).

Finalmente, la variación genética es un requerimiento básico para el cultivo $\mathrm{y}$ especialmente para el proceso de mejoramiento genético de plantas; mientras que la alta variación genética es necesaria para el fitomejoramiento (Kameli et al., 2013). La exploración y evaluación de la variabilidad entre y dentro de la población podría ser de gran significancia para la conservación in situ.

El estudio de los polimorfismo determinado por marcadores moleculares en este caso ISSR (que son altamente reproducibles), es un parámetro muy valioso para estudiar y entender las diferencias genéticas en las poblaciones.

\section{Tabla 4}

Polimorfismo de productos de amplificación de las 3 accesiones de L. mutabilis. Donde PIC: Índice de contenido polimórfico y \%P: porcentaje de loci polimórfico

\begin{tabular}{|c|c|c|c|c|c|c|c|c|c|c|c|c|}
\hline \multicolumn{3}{|c|}{ Primers } & 811 & 834 & 835 & 841 & 842 & 889 & 891 & Bor5 & \multirow{3}{*}{ Total } & \multirow{3}{*}{$\mathrm{X}$} \\
\hline & & & $3000 \leq x$ & $3000 \leq x$ & $3000 \leq x$ & $3000 \leq x$ & $3000 \leq x$ & $3000 \leq x$ & $3000 \leq x$ & $3000 \leq x$ & & \\
\hline \multicolumn{3}{|c|}{ Accesiones } & $\geq 900 \mathrm{pb}$ & $\geq 400 \mathrm{pb}$ & $\geq 400 \mathrm{pb}$ & $\geq 600 \mathrm{pb}$ & $\geq 300 \mathrm{pb}$ & $\geq 900 \mathrm{pb}$ & $\geq 400 \mathrm{pb}$ & $\geq 500 \mathrm{pb}$ & & \\
\hline \multirow{3}{*}{ Ta05 } & \multicolumn{2}{|c|}{$\mathrm{N}^{\circ}$ de bandas } & 17 & 21 & 31 & 32 & 24 & 17 & 31 & 16 & 189 & 24 \\
\hline & Bandas & $\mathrm{N}^{\circ}$ & 9 & 14 & 14 & 13 & 10 & 6 & 14 & 7 & 87 & 11 \\
\hline & polimórficas & $\%$ & 52,94 & 66,67 & 45,16 & 40,63 & 41,67 & 35,29 & 45,16 & 43,75 & 46,03 & - \\
\hline \multirow{2}{*}{ Ta07 } & \multicolumn{2}{|c|}{$\mathrm{N}^{\circ}$ de bandas } & 17 & 28 & 30 & 27 & 29 & 17 & 27 & 15 & 190 & 24 \\
\hline & polimórficas & $\%$ & 58,82 & 50 & 43,33 & 29,63 & 41,38 & 41,18 & 44,44 & 60,00 & 44,74 & - \\
\hline \multirow{3}{*}{ Ta09 } & \multicolumn{2}{|c|}{$\mathrm{N}^{\circ}$ de bandas } & 14 & 24 & 33 & 31 & 32 & 16 & 24 & 12 & 186 & 23 \\
\hline & \multirow{2}{*}{$\begin{array}{c}\text { Bandas } \\
\text { polimórficas }\end{array}$} & $\mathrm{N}^{\circ}$ & 8 & 14 & 11 & 10 & 15 & 10 & 10 & 4 & 82 & 10 \\
\hline & & $\%$ & 57,14 & 58,33 & 33,33 & 32,26 & 46,88 & 62,5 & 41,67 & 33,33 & 44,09 & - \\
\hline \multicolumn{3}{|c|}{$\mathrm{P} \%$ promedio } & 56,3 & 58,33 & 40,61 & 34,17 & 43,31 & 46,32 & 43,76 & 45,69 & 44,95 & \\
\hline
\end{tabular}




\section{Conclusiones}

La variabilidad genética inter-accesión de L. mutabilis, según las accesiones $\mathrm{y}$ marcadores ISSR evaluados, es considerable. Por lo que esta investigación demuestra que L. mutabilis es una planta autógama con un considerable grado de alogamia, lo que facilita su mejoramiento genético. El uso de iniciadores ISSR para este cultivo es fiable ya que los resultados han mostrado la coherencia del fenograma, basado en el análisis de similitud, con los datos pasaporte y la ubicación geográfica. Los iniciadores ISSR 834 y 835 son los más polimórficos en el estudio tanto dentro (intragenotípico) como entre (intergenotípico) las accesiones; mientras que, los iniciadores ISSR 834, 811, 841, 835 y BOR5 presentar mayor contenido de información polimórfica en el estudio de variabilidad genética intra-accesión.

\section{Agradecimientos}

Al Programa de Cereales y Granos Nativos de la Universidad Nacional Agraria La Molina por el financiamiento de los materiales y reactivos usados en la investigación.

\section{Referencias bibliográficas}

Aguilera, J.G.; Pessoni, L.A.; Rodrigues, G.B., Elsayed, A.Y.; Da Silva, D.J.; De Barros, E.G. 2011. Genetic variability by ISSR markers in tomato (Solanum lycopersicon Mill). Revista Brasileira Ciencias Agrarias 6: 243-252.

Astarini, I.A.; Plummer, J.A.; Lancaster, R.A.; Yan, G. 2006. Genetic diversity of Indonesian cauliflower cultivars and their relationships with hybrid cultivars grown in Australia. Scientia Horticulturae 108: 143150.

Azofeifa, A. 2006. Uso de marcadores moleculares en plantas: aplicaciones en frutales del trópico. Agronomía Mesoamericana 17: 221-242.

Bornet, B.; Branchard, M. 2001. Nonanchored inter simple sequence repeat (ISSR) markers: reproducible and specific tools for genome fingerprinting. Plant Molecular Biology Reporter 19: 209-215.

Bussell, J.D.; Waycott, M.; Chappill, J.A. 2005. Arbitrarily amplified DNA markers as characters for phylogenetic. Perspectives in Plant Ecology, Evolution and Systematics 7: 3-26.

Camarena F, Huaringa A, Jiménez J, Mostacero E. 2012. Revalorización de un cultivo subutilizado: Chocho o Tarwi (Lupinus mutabilis Sweet). Editorial CONCYTEC, Lima, Perú.

Cao, P.Y.; Yao, Q.F.; Ding, B.Y.; Zeng, H.Y.; Zhong, Y.X.; Fu, C.X.; Jin, X.F. 2006. Genetic diversity of Sinojackia dolichocarpa (Styracaceae), a species endangered and endemic to China, detected by inter- simple sequence repeat (ISSR). Biochemical Systematics and Ecology 34: 231-239.

Castañeda, B.; Manrique, R.; Gamarra, F.; Muñoz, A.; Ramos, F.; Lizaraso, F.; Martínez, J. 2008. Probiótico elaborado en base a las semillas de Lupinus mutabilis Sweet. Acta Médica Peruana 25: 210-215.

Castañeda, B.; Castro de la Mata, R.; Manrique, E.; Ibañez, L. 2007. Efectos metabólicos de Lepidium meyenii Walpers."MACA" y Lupinus mutabilis Sweet. "chocho" en ratas. Revista Horizonte Médico 7: 32-38.

Ciancio, A.; Mukerji, K.G. 2008. Integrated Management and Biocontrol of Vegetable and Grain Crops Nematodes 2: 102-103

Doyle, J.J.; Doyle, J.L. 1987. A rapid DNA isolation procedure from small quantities of fresh leaf tissues. Phytochem Bull. 19:11-15.

Eguiarte, L.E.; Souza, V.; Aguilar, X. 2007. Ecología Molecular. Editorial SEMARNAT. México.

FAOa. Org. Comunicado de prensa: 925 millones de personas sufren hambre crónica en el mundo. Disponible http://www.fao.org/news/story/es/item/45291/icode/

FAOb. Org. Cultivos Andinos, tarwi o chocho (Lupinus mutabilis). Disponible en: http://www.rlc.fao.org/es/agricultura/produ/cdrom/cont enido/libro10/cap03_13

Ferriol, M.; Picó, B.; Nuez, F. 2001. Genetic Variability in Pumpkin (Cucurbita maxima) Using RAPD Markers. Cucurbit Genetics Cooperative Report 24: 94-96.

Fikiru, E.; Tesfaye, K.; Bekele, E. 2007. Genetic diversity and population structure of Ethiopian lentil (Lens culinaris Medikus) landraces as revealed by ISSR marker. African J. of Biotechnology 6: 1460-1468.

Fisher, P.J.; Gardner, R.C. y Richardson, T.E. 1996. Single locus microsatellites isolated using 5'anchored PCR. Nucleic Acids Research 24: 4369-4371.

Gilbert, J.E.; Lewis, R.V.; Wilkinson, M.J.; Caligari; P.D.S. 1999. Developing an appropriate strategy to assess genetic variability in plant germplasm collections. Theor. Appl. Genet. 98: 1125-1131.

Girma, G.; Tesfaye, K.; Bekele, E. 2010. Inter Simple Sequence Repeat (ISSR) analysis of wild and cultivated rice species from Ethiopia. African Journal of Biotechnology 9: 5048-5059.

Gross, R.; von Baer, E.; Koch, F.; Marquard, R.; Trugo, L.; Wink, M. 1988. Chemical composition of a new variety of the Andean lupin (Lupinus mutabilis cv. Inti) with low alkaloid content. J. Food Comp. Anal. 1: 353-361.

Guo, H.B.; Huang, K.Y.; Zhou, T.S.; Wu, Q.H.; Zhang, Y.J.; Liang, Z.S. 2009. DNA isolation, optimization of ISSR-PCR system and primers screening of Scutellaria baicalensis. Journal of Medicinal Plants Research 3(11): 898-901.

Hatcher, P.E.; Wilkinson, M.J.; Albani, M.C.; Hebbern, C.A. 2004. Conserving marginal populations of the food plant (Impatiens noli-tangere) of an endangered moth (Eustroma reticulatum) in a changing climate. Biological Conservation 116: 305-317.

Hollingsworth, P.M.; Ennos, R.A. 2004. Neighbour joining trees, dominant markers and population genetic structure. Heredity 92: 490-498.

Horovitz, A.; Harding, J. 1983. Genetics of Lupinus. XII. The mating system of Lupinus pilosus. Bot. Gaz. 144: 276-279.

Hou, Y.C.; Yan, Z.H.; Wei, Y.M.; Zheng, Y.L. 2005. Genetic diversity in barley from west China based on RAPD and ISSR analysis. Barley Genetics Newsletter 35: 9-22. 
Isshiki, S.; Iwata, N., Mizanur, M.D. 2008. ISSR variations in eggplant (Solanum melongena L.) and related Solanum species. Scientia Horticulturae 117: 186-190.

Jacobsen, E.; Mujica, A. 2006. El tarwi (Lupinus mutabilis Sweet) y sus parientes silvestres. Universidad Mayor de San Andrés, La Paz, Bolivia. Pp. 458-482.

Jian, S.; Tang, T.; Zhong, Y.; Shi, S. 2004. Variation in inter-simple sequence repeat (ISSR) in mangrove and non-mangrove populations of Heritiera littoralis (Sterculiaceae) from China and Australia. Aquatic Botany 79: 75-86.

Jiménez, J. 2006. Biodiversity of traditional seed propagated crops cultivated in Peruvian highland. Tesis Ph.D. University of SILESIA. Polonia.

Joshi, N.; Rawat, A.; Subramanian, R.B.; Rao, K.S. 2010. A method for small scale genomic DNA isolation from chickpea (Cicer arietinum L.) suitable for molecular marker analysis. Indian Journal of Science and Technology 3: 0974- 6846.

Kameli, M.; Hesamzadeh, S.M.; Ebadi, M. 2013. Assessment of genetic diversity on populations of three satureja species in Iran using ISSR markers. Annals of Biological Research 4:64-72 63

Konate, I.; El-Bekkay, B.; Filali-Maltouf, A. 2009. Intersimple sequence repeat markers variation among natural accessions of Moroccan carob tree (Ceratonia siliqua). Int. J. Agric. Biol. 11: 168-172.

Kothera, L.; Ward, S.M.; Carney, S.E. 2007. Assessing the threat from hybridization to the rare endemic Physaria belli Mulligan (Brassicaceae). Biological Conservation 140: 110-118. 65.

Lázaro, A.; Aguinagalde, I. 2006. Genetic variation among Spanish pea landraces revealed by Inter Simple Sequence Repeat (ISSR) markers: its application to establish a core collection. Journal of Agricultural Science 144: 53-61.

Liu, L.W.; Zhao, L.P; Gong, Y.Q.; Wang, M.X.; Chen, L.M.; Yang, J.L; Wang, Y.; Yu, F.M.; Wang, L.Z. 2008. DNA fingerprinting and genetic diversity analysis of late-bolting radish cultivars with RAPD, ISSR and SRAP markers. Sci. Hort. 116: 240- 247.

Loarce, Y.; Gallego, R.; Ferrer, E. 1996. A comparative analysis of the genetic relationships between rye cultivars using RFLP and RAPD markers. Euphytica 88: 107-115.

Moreno, Y.; Melgarejo, L.M; Hernández, M.S.; Quintero, L.; Vargas, G. 2004. Caracterización molecular de un banco de germoplasma del género Theobroma mediante marcadores RAPD. Revista Colombiana de Biotecnología 4(2): 15-24.

Mujica, A. 1990. Investigación y producción del tarwi (Lupinus mutabilis Sweet.) en el Perú. INIAA, PICA. Lima.

Muthusamy, S.; Kanagarajan, S., Ponnusamy, S. 2008. Efficiency of RAPD and ISSR markers system in accessing genetic variation of rice bean (Vigna umbellata) landraces. Electronic Journal of Biotechnology 11: 0717-3458.

Nascimiento, M.A.; Batalha-Filho, H.; Waldschmidt, A.M.; Tavares, M.G.; Campos, L.A.O; Salomão, T.M. 2010. Variation and genetic structure of Melipona quadrifasciata Lepeletier (Hymenoptera, Apidae) populations base on ISSR pattern. Genetics and Biology 33(2): 394-397.

Ovesná, J.; Poláková, K.; Leisová, L. 2002. DNA Analyses and their Applications in Plant Breeding. Czech J. Genet. Plant Breed. 38: 29-40.

Palacios, A. 2003. Obtención de alcohol a partir de la malta de Lupinus mutabilis (Tarwi). Facultad de Ingeniería química. Universidad Nacional del Centro del Perú.

Rakoczy M.; Bolibok H. 2004. Characteristics and comparison of three classes of microsatellite-based markers and their application in plants. Cellular and molecular Biology Letters 9: 231-238.

Roh, M.S.; Cheong, E.J.; Choi, I.K.; Joung, Y.H. 2007. Characterization of wild Prunus yedoensis analyzed by inter-simple sequence repeat and chloroplast DNA. Scientia Horticulturae 114: 121-128.

Sbabou, L.; Brhada, F.; Alami, I.T. 2010. Genetic Diversity of Moroccan lupinus Germplasm Investigated using ISSR and AFLP Markers. International Journal of Agricultural \& Biology 12: 26-32.

Sevilla, R.; Holle, M. 2004. Formas de reproducción de las plantas y Caracterización del germoplasma. Editorial Recursos genéticos vegetales Luis león Asociados S. R. L., Lima, Perú.

Shafie, M.; Hasan, S., Zain, A.; Shah, R. 2011. RAPD and ISSR markers for comparative analysis of genetic diversity in wormwood capillary (Artemisia capillaris) from Negeri Sembilan, Malaysia. J. Med. Plant. Res. 5(18): 4426-4437.

Sica, M.; Gamba, G.; Montieri, S.; Gaudio, L.; Aceto, S. 2005. ISSR markers show differentiation among Italian populations of Asparagus acutifolus L. BMC Genet. 6: 17

Su, Y.J.; Zan, Q.J.; Wang, T.; Ying, Z.M.; Ye, H.G 2008. High ISSR variation in 24 surviving individuals of Apterosperma oblata (Theaceae) endemic to China. Biochemical Systematics and Ecology 36: 619-625.

Svetleva, D.; Pereira, G.; Carlier, J.; Cabrita, L.; Leitão, J.; Genchev, D. 2006. Molecular characterization of Phaseolus vulgaris L. genotypes included in Bulgarian collection by ISSR and AFLP analyses. Sci Hort 109: 198-206.

Talhinhas, P.; Neves-Martins, J.; Leitao, J. 2003. AFLP, ISSR and RAPD markers reveal high levels of genetic diversity among Lupinus spp. Plant Breeding 122: 507-510.

Venkatachalam, L.; Sreedhar, R.V.; Bhagyalakshmi, N. 2008. The use of genetic markers for detecting DNA polymorphism, genotype identification and phylogenetic relationships among banana cultivars. Molecular Phylogenetics and Evolution 47: 974-985.

Vuylsteke, M.; Mank, R.; Brugmans, B.; Stam, P.; Kuiper, M. 2000. Further characterization of AFLP data as a tool in genetic diversity assessments among maize (Zea mays) inbred lines. Mol. Breed. 6: 265-276.

Wang, C.; Zhang, H.; Qian, Z.Q.; Zhao, G.F. 2008. Genetic differentiation in endangered Gynostemma pentaphyllum (Thunb.) Makino based on ISSR polymorphism and its implications for conservation. Biochemical Systematics and Ecology 36: 699-705.

Weising, K.; Nybom, H., Pfenninger, M.; Wolff, K.; Kahl, G. 2005. DNA fingerprinting in Plants. Principles, Methods, and Applications. Taylor and Francis Group, Boca Ratón. Pp 444.

$\mathrm{Xu}$, F.; Sun, M. 2001. Comparative analysis of phylogenetic relationships of grain Amaranths and their wild relatives (Amaranthus; Amaranthaceae) using internal transcribed spacer, Amplified Fragment Length Polymorphism and Double-primer Fluorescent Intersimple Sequence Repeat Markers. Molecular Phylogenetics and Evolution 21: 372-387.

Yang, X.; Quiros, C. 1993. Identification and classification of celery cultivars with RAPD markers. Theor. Appl. Genet. 86: 205-212. 
Zietkiewicz, E.; Rafalski, A.; Labuda, D. 1994. Genome fingerprinting by simple sequence repeats (SSR)anchored polymerase chain reaction amplification. Genomics 20: 176-183.

Zhao, J.; Wang, X.; Deng, B.; Lou, P.; Wu, J.; Sun, R.; Xu, Z.; Vromans, J.; Koornneef, M.; Bonnema, G. 2005. Genetic relatioships within Brassica rapa as inferred from AFLP fingerprints. Theor. Appl. Genet. 110: 1301-1314.
Zhu, Y.; Hu, J.; Han, R.; Wang, Y.; Zhu, S. 2011. Fingerprinting and identification of closely related wheat (Triticum aestivum L.) cultivars using ISSR and fluorescence-labeled TP-M13-SSR markers. Australian Journal of Crop Science 5: 846-850. 